\title{
Uptodate sul calcium-sensing receptor
}

\author{
Teresa Arcidiacono ${ }^{1}$, Annalisa Terranegra ${ }^{2}$, Alessandra Mingione $^{2}$, Laura Soldati², \\ Lorenza Macrina ${ }^{1}$, Giuseppe Vezzoli ${ }^{1}$
}

${ }^{1}$ Unità di Nefrologia e Dialisi, Università Vita-Salute, Ospedale San Raffaele, Milano

${ }^{2}$ Dipartimento di Scienze della Salute, Università degli Studi di Milano, Ospedale San Paolo, Milano

\begin{abstract}
UPDATE ON THE CALCIUM-SENSING RECEPTOR
Abstract. The cells of the human body have a receptor that is sensitive to extracellular calcium [calcium-sensing receptor (CaSR)] and whose tissue expression is decreased in patients with chronic kidney disease. The CaSR plays a key role both in the regulation of PTH secretion by parathyroid cells, as well as in calcium tubular reabsorption by renal tubular cells. Recent biochemical and cellular studies found that this receptor has peculiar functional characteristics. Furthermore, pharmacological research has brought to the marker agonists for CaSR (calcimimetics) that today are used for treating secondary hyperparathyroidism and may have an effect on cardiovascular and bone diseases in patients with chronic kidney disease. With regard to the latter, the EVOLVE and ADVANCE trials were dedicated to understanding the role of cinacalcet, a calcimimetic drug, in the prevention of vascular calcification and cardiovascular events in patients with chronic kidney disease. Research in this field has thus opened promising perspectives for the treatment of chronic kidney disease.
\end{abstract}

Key words: Calcium-sensing receptor, Hyperparathyroidism

Conflict of interest: None.

Ricevuto: 18 Luglio 2013; Accettato: 31 Luglio 2013

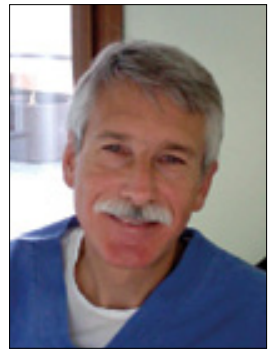

Giuseppe Vezzoli

\section{Introduzione}

Le cellule dell'organismo umano possiedono sulla loro membrana plasmatica un recettore sensibile al calcio (calcium sensing receptor, $\mathrm{CaSR}$ ), che trova la sua massima intensità di espressione nelle cellule della paratiroide e del tubulo renale (1). La presenza del CaSR consente alle cellule di modificare la propria attività in base ai valori del calcio ematico. In particolar modo, consente alla paratiroide e al tubulo distale renale di regolare rispettivamente la secrezione del PTH e il riassorbimento del calcio, modulandoli sulla base della concentrazione di calcio ematico $(2,3)$. Il CaSR è una proteina di $1078 \mathrm{D}$, che appartiene alla famiglia dei recettori accoppiati alle proteine G (GPCR), con i quali condivide la struttura e le vie di segnalazione intracellulare. Viene codificato dal cromosoma 3q13.3-21 e il suo gene presenta 2 promotori e 7 esoni, il primo dei quali non tradotto (1, 2). La sua molecola è composta da un dominio extracellulare, un dominio transmembrana e un dominio intracellulare (Fig. 1). Il primo lega il calcio extracellulare, mentre gli altri due attivano le vie di signalling intracellulari, interagendo con le proteine $\mathrm{G}$ e la filamina A (1). I sistemi di signalling comprendono anche le proteinchinasi attivate dai mitogeni (MAPK) e diversi fattori di crescita (4). Tra questi ultimi è stata recentemente osservata l'attivazione di fattori di trascrizione genica come Egr-1 (early growth response protein 1), Elk-1 (ets-like protein-1) e Ap-1 (activator protein-1). Questi dati dimostrano come la regolazione dell'espressione genica sia parte integrale del sistema di signalling indotto dal CaSR nelle cellule (5).

\section{Aspetti funzionali e biochimici}

Dal punto di vista biochimico, il CaSR presenta caratteristiche funzionali particolari che lo differenziano dagli altri recettori cellulari. Questi instaurano un legame ad alta affinità con $i$ loro agonisti, che ben si adatta al range di concentrazione plasmatica di questi ultimi, usualmente picomolare. Il legame con l'agonista attiva l'internalizzazione e l'inattivazione dei recettori di membrana allo scopo di limitarne gli effetti (1-3). Nel caso del CaSR, il principale agonista è lo ione calcio, che ha concentrazioni intorno a $1.25 \mathrm{mmol} / \mathrm{L}$ nel fluido extracellulare umano. Il CaSR lega, però, il calcio con bassa affinità, nell'ordine millimolare (EC50 in vitro di circa $3 \mathrm{mmol} / \mathrm{L}$ ), e questo fa 


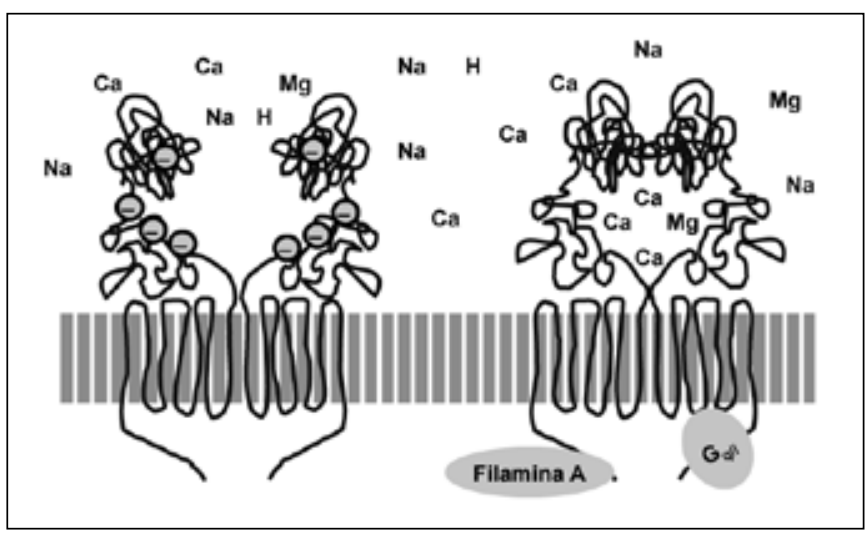

Fig. 1 - Il CaSR è una proteina di 1078 D, espressa come dimero sulla membrana plasmatica nelle cellule dell'organismo umano. È composto da un dominio extracellulare (612 amminoacidi), un dominio transmembrana ( 250 amminoacidi) e un dominio intracellulare (216 amminoacidi). Il dominio extracellulare della molecola forma una tasca ricca di cariche negative che legano i cationi presenti nel sangue. Quando avviene il legame, il dominio extracellulare si ripiega su se stesso, trasmettendo il segnale agli altri domini che attivano le vie di signalling intracellulari interagendo con le proteine $\mathrm{G}$ e la filamina $\mathrm{A}$.

del calcio il principale agonista del CaSR. Queste caratteristiche funzionali consentono al CaSR di svolgere il suo ruolo chiave nell'omeostasi del metabolismo minerale e al calcio di possedere il ruolo funzionale che riveste. Il CaSR non lega solo il calcio, ma tutti i cationi: quelli trivalenti sono legati con maggiore affinità rispetto al calcio, ma, essendo presenti nel plasma solo in tracce, non possono rivestire un ruolo funzionale; quelli monovalenti sono legati con affinità inferiore rispetto al calcio (1). Un particolare aspetto della funzione del CaSR è la sua persistente capacità di rispondere al calcio nella cellula, nonostante la stabilità dell'elevata concentrazione ematica del calcio, che dovrebbe teoricamente provocare l'inattivazione del CaSR e la sua proteolisi lisosomiale $(6,7)$. La cinetica cellulare del CaSR è, però, del tutto particolare, perché una quantità significativa di molecole di CaSR viene conservata nel reticolo endoplasmatico e nell'apparato di Golgi (Fig. 2). La sua traslocazione sulle membrane cellulari viene stimolata dall'incremento della calcemia mediante un blocco della glicosilazione delle molecole intracellulari. Questo fenomeno è stato descritto per la prima volta nel 2011 ed è un nuovo sistema di signalling, denominato signalling inserzionale attivato dall'agonista (ADIS), specifico del CaSR e capace di garantirne la presenza sulla membrana per mantenere la risposta cellulare al calcio (6).

\section{CaSR e metabolismo del calcio renale}

La presenza del CaSR consente alle cellule principali della paratiroide di regolare la secrezione del PTH in funzione della calcemia. Esso, perciò, garantisce il set-point della paratiroide, affinché la ghiandola possa mantenere la calcemia nel range di norma (2). Il rene esprime maggiormente il CaSR nelle cellule tubulari, soprattutto sulla membrana basolaterale del tratto

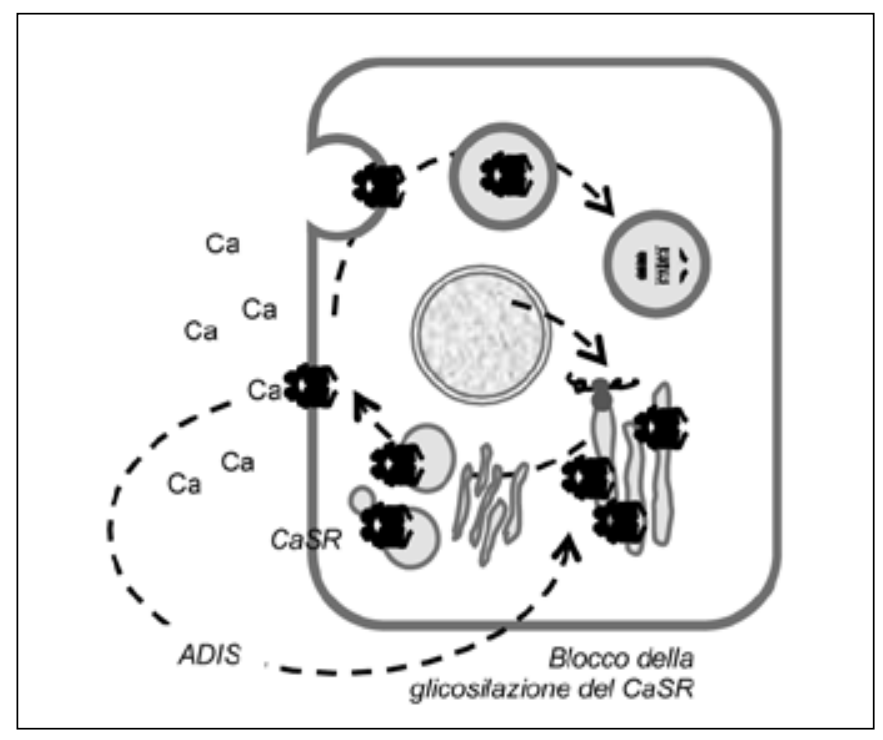

Fig. 2 - A differenza degli altri recettori, una quantità significativa di molecole di CaSR viene conservata nel reticolo endoplasmatico e nell'apparato di Golgi. La traslocazione sulle membrane cellulari viene stimolata dall'incremento della calcemia mediante un blocco della glicosilazione delle molecole intracellulari. Questo fenomeno è denominato ADIS (agonist-driven insertional signalling) ed è capace di garantire la presenza del CaSR sulla membrana mantenendo la risposta cellulare al calcio nonostante la sua elevata e stabile concentrazione ematica (intorno a 1.25 $\mathrm{mmol} / \mathrm{L}$ ), che dovrebbe, teoricamente, provocare la continua inattivazione del CaSR e la sua proteolisi lisosomiale $(6,7)$.

ascendente spesso dell'ansa di Henle. In questa sede, l'attivazione del CaSR da parte del calcio ematico diminuisce il riassorbimento passivo del calcio (3). Nel tubulo convoluto distale, immediatamente successivo al tratto ascendente spesso, l'attivazione del CaSR inibisce, invece, il riassorbimento attivo di calcio (3). È stato recentemente dimostrato che gli effetti tubulari del CaSR abilitano il rene al controllo della calcemia (8). L'esperimento più significativo a tale riguardo è stato eseguito su ratti paratiroidectomizzati e trattati con un bisfosfonato (pamidronato) per bloccare il riassorbimento osseo. In questi ratti la somministrazione di un farmaco calciolitico (NPS2143) faceva diminuire l'escrezione di calcio e aumentare la concentrazione sierica del calcio ionico. Questo dimostrava che, attraverso il CaSR, il riassorbimento tubulare renale poteva controllare la calcemia in modo autonomo dalla paratiroide (8).

L'effetto del CaSR sul riassorbimento di calcio nel tratto ascendente spesso avviene in modo complesso. In questo tratto, il cotrasportatore sodio-potassio-cloro (NKCC2) media il riassorbimento di due ioni cloro insieme a uno ione sodio e a uno potassio attraverso la membrana apicale. Lo ione potassio, una volta riassorbito, può ritornare nel lume tubulare attraverso il canale Kir1.1 (ROMK), generando un potenziale transepiteliale positivo dal lume tubulare all'interstizio. Questo gradiente elettrico diventa la forza per il riassorbimento passivo paracellulare del calcio e degli altri cationi (9).

E stato recentemente scoperto che il riassorbimento paracel- 


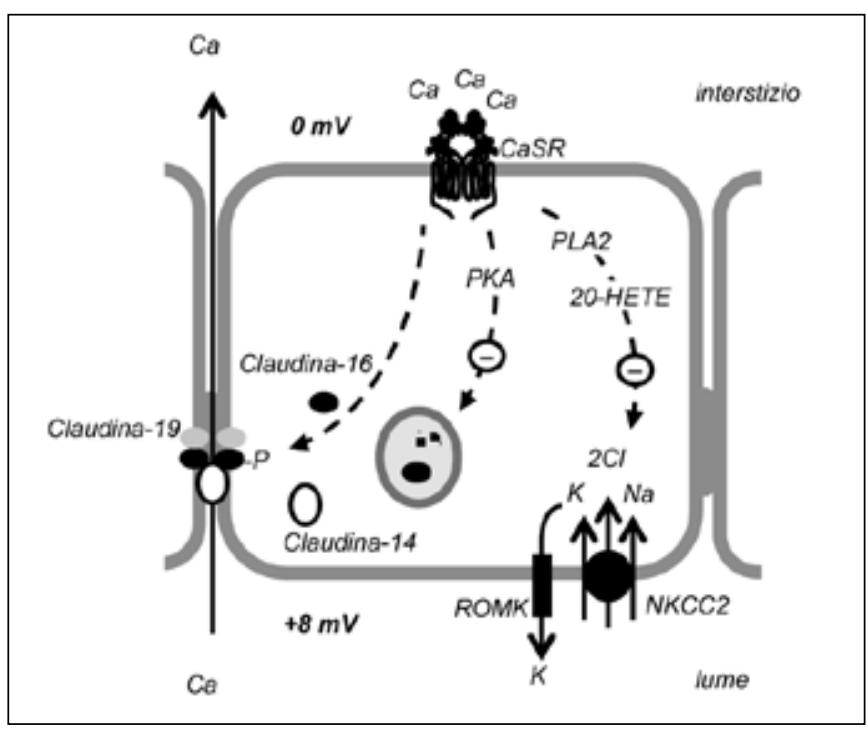

Fig. 3 - Il riassorbimento paracellulare del calcio nel tratto ascendente spesso dell'ansa avviene attraverso canali specifici per gli ioni bivalenti posti nelle tight-junction e composti dalla claudina-16 e -19, ai quali si aggiunge la claudina-14 come proteina che chiude il canale. L'espressione della claudina-16 nelle tight-junction dipende dalla sua fosforilazione che viene inibita dal CaSR attraverso l'attivazione della fosforilasi chinasi $\mathrm{A}$ (PKA). Anche l'espressione della claudina-14 nelle tight-junction dipende dal CaSR e viene stimolata anche dalla dieta ricca di calcio. Questi effetti, nel loro insieme, riducono il riassorbimento passivo di calcio in questo tratto, nel quale il riassorbimento viene guidato dal potenziale di membrana generato dal riassorbimento di sodio, potassio e cloro attraverso $\mathrm{NKCC} 2$, al quale fa seguito la secrezione di potassio attraverso ROMK, che fa diventare elettrogenico questo sistema di trasporto (10, 11). Anche su questi trasporti il CaSR ha un effetto di inibizione attraverso la produzione di 20-eicosatetraenoico (20-HETE), secondaria all'attivazione della fosfolipasi A2 (PLA2).

lulare di calcio nel tratto ascendente utilizza dei canali posti nelle tight-junction (giunzioni strette). Questi canali sono formati dalla claudina-16 e -19 (Fig. 3), alle quali si aggiunge la claudina-14, specificatamente espressa nel tratto ascendente spesso, che, però, determina una riduzione della conduttanza del canale e del riassorbimento paracellulare di calcio e di magnesio $(10,11)$. È stato anche osservato come l'espressione della claudina-14 fosse positivamente correlata all'apporto di calcio con la dieta e come questa dipendenza fosse probabilmente mediata dal CaSR. Due microRNA (miR-9 e miR-374) potrebbero stimolare l'espressione renale di claudina-14 a seguito della dieta ricca di calcio. A supporto di queste osservazioni, i topi sottoposti a delezione del gene CaSR nel loro rene diminuivano l'espressione renale di claudina-14 e -16, ma non quella della claudina-19. Inoltre, in questi topi era aumentata anche l'attività del carrier $\operatorname{NKCC} 2(10,11)$.

\section{CaSR e metabolismo osseo}

Il ruolo del CaSR nelle cellule ossee è stato studiato mediante delezione genetica selettiva in singole tipologie cellulari. I topi con specifica delezione del gene CaSR nella paratiroide, oltre a sviluppare iperparatiroidismo, presentavano un ritardo dello sviluppo dello scheletro, che appariva gravemente demineralizzato e ricco di tessuto osteoide (12). Le trabecole ossee risultavano diminuite e il tessuto corticale meno spesso. Conseguenze di questi danni erano la ridotta resistenza scheletrica e la tendenza alla fratturazione. La diminuita espressione ossea di collagene I (Col-1), fosfatasi alcalina (ALP), proteina 1 della matrice della dentina (DMP1), osteocalcina (OCN), sclerostina (SOST) e fattore di crescita insulinico 1 (IGF-1) dimostrava una ridotta funzione osteoblastica che poteva essere responsabile delle alterazioni ossee descritte.

I topi con delezione del gene CaSR nei soli osteoblasti presentavano un deficit della crescita e dello sviluppo delle ossa, che apparivano dimensionalmente più piccole e demineralizzate sia a livello trabecolare che corticale. L'analisi dello scheletro mostrava ridotta massa ossea, accumulo di tessuto osteoide e deficit della microarchitettura ossea in diversi segmenti scheletrici. La differenziazione osteoblastica era ritardata ed era scarsa l'espressione dei fattori osteogenici Col-1, ALP, DMP1, OCN, SOST e IGF-1 (12). Un ulteriore contributo è stato recentemente fornito da uno studio di topi privati del gene CaSR a livello osteoblastico (13). Questo studio, oltre a confermare i dati appena descritti, ha osservato un aumento dell'espressione ossea di fattori rilevanti per la mineralizzazione ossea, tra i quali l'osteopontina, e del recettore attivante il fattore nucleare-kB (RANKL). Quest'ultimo è prodotto dagli osteoblasti ed è il principale stimolatore della differenziazione dei preosteoclasti in osteoclasti e della loro attività. Lo studio istomorfometrico del femore distale e medio di questi topi ha evidenziato un aumento del numero degli osteoclasti e della percentuale della superficie di erosione. Gli Autori dello studio hanno, perciò, concluso che il CaSR degli osteoblasti è essenziale per lo sviluppo e il rimodellamento osseo, perché gioca un ruolo importante nel riassorbimento e nella mineralizzazione ossea (13).

\section{CaSR, calciolitici e osteoporosi}

La scoperta del CaSR (1993) ha aperto la strada a nuovi farmaci che, influenzando la sua attività, sono potenzialmente utilizzabili per le malattie del metabolismo calcio-fosforo e dello scheletro. I calciomimetici sono attivatori allosterici che incrementano l'affinità di legame tra il CaSR e gli agonisti. I calciolitici sono, viceversa, farmaci che diminuiscono l'attività del CaSR agli agonisti. Nel ratto ovariectomizzato ed osteopenico, la somministrazione del calciolitico NPS-2143 causava secrezione tonica di PTH, con un picco sierico dopo poco meno di un'ora dalla somministrazione del farmaco. Dopo settimane di trattamento si assisteva all'aumento della massa ossea e del numero e dello spessore delle trabecole ossee (14). Alla luce di questi dati, è stato utilizzato il calciolitico ronacaleret per trattare l'osteoporosi postmenopausale. È stato condotto un trial randomizzato e controllato con placebo che paragonava l'effetto di ronacaleret, teriparatide e alendronato sulla massa minerale e sui marker del turnover osseo. Il ronacaleret era somministrato a dosi da 10 a $300 \mathrm{mg} /$ die e le pazienti $(n=569)$ erano seguite lungo un follow-up di 
12 mesi. I risultati del trial sono stati, però, negativi perché la massa minerale non aumentava, sia quando misurata con metodo DEXA areale in sede lombare e femorale (15) sia quando misurata con metodo pQCT volumetrico. Con il metodo pQCT era osservato un incremento modesto della massa trabecolare, controbilanciato da una diminuzione della massa corticale sia in sede lombare che in sede femorale. Il motivo dell'insuccesso è stato attribuito al fatto che il calciolitico non provocava picchi sierici del PTH, ma una condizione di lieve iperparatiroidismo, perché i livelli di PTH persistevano elevati fino ad 8 ore dopo la sua somministrazione $(16,17)$.

\section{CaSR e malattia cardiovascolare nella CKD}

Nella malattia renale cronica (CKD) si instaura precocemente una diminuzione dell'espressione tissutale del CaSR, che contribuisce all'insorgenza del danno osseo e vascolare, cioè della "metabolic bone disease" (18).

La riduzione dell'espressione tissutale del CaSR è di primaria importanza nelle ghiandole paratiroidi, nelle quali è direttamente coinvolta nella patogenesi dell'iperparatiroidismo secondario alla CKD. Il deficit di CaSR comporta la ridotta capacità di inibire la secrezione del PTH da parte del calcio sierico e il conseguente incremento del set-point e del volume della ghiandola paratiroide. La causa della ridotta espressione del CaSR risiede nella carenza di 1.25-diidrossicolecalciferolo $\left(1,25 \mathrm{OH}_{2} \mathrm{D}\right)$ e del suo recettore VDR, tipica della CKD. Il gene CaSR è, infatti, fornito di due promotori che possiedono la sequenza di legame specifica per il complesso $1.25 \mathrm{OH}_{2} \mathrm{D}$ VDR, che può attivare, così, la trascrizione (19).

Non sono disponibili studi che abbiano valutato l'espressione del CaSR nelle cellule ossee dei pazienti uremici, ma, in considerazione dell'importanza del CaSR per la funzione degli osteoblasti, si può ipotizzare che la sua carenza possa essere un fattore determinante per lo sviluppo di un osso adinamico spesso osservato nei pazienti con CKD.

La carenza di CaSR è stata osservata anche nella parete arteriosa dei pazienti con CKD. In questa sede, il CaSR viene espresso sia dalle cellule endoteliali che da quelle muscolari lisce. Nelle prime stimola la produzione di ossido nitrico tramite l'attivazione della sintasi specifica (20). Il deficit di CaSR nell'endotelio potrebbe, perciò, rivestire un ruolo importante per la regolazione del flusso ematico nel microcircolo, nonché della pressione arteriosa, perché potrebbe limitare la vasodilatazione mediata dall'ossido nitrico. Oltre a questo, il CaSR può stimolare la vasodilatazione attraverso la produzione di sostanze vasodilatatrici da parte delle terminazioni nervose sensoriali perivascolari (21). Un terzo fattore di vasodilatazione potrebbe dipendere dalla capacità del CaSR di liberare ioni calcio dal reticolo endoplasmatico delle cellule muscolari lisce della parete arteriosa, alla quale farebbe seguito il rilassamento cellulare (22). Il deficit di CaSR nelle arterie potrebbe, perciò, essere accompagnato da un'inadeguata regolazione del tono vascolare secondaria alla carenza di fattori vasodilatatori. La carenza di CaSR nella parete arteriosa è stata implicata nella calcificazione della parete vascolare, che, nei pazienti con CKD, si sviluppa nella tonaca media. È questo un importante fattore di rischio cardiovascolare, connesso con le al- terazioni del metabolismo calcio-fosforo e, particolarmente, con l'iperfosforemia (23). Il ruolo del CaSR nel processo di formazione delle calcificazioni arteriose non è stato ancora capito; è, però, stato osservato che la somministrazione di calciomimetici previene la calcificazione delle arterie nell'animale uremico $(24,25)$.

Questi dati hanno fatto riporre molte speranze nelle potenzialità terapeutiche dei calciomimetici per la cura dell'osteodistrofia e della vasculopatia del paziente nefropatico. Lo studio EVOLVE ha affrontato tutti questi aspetti in una casistica di 3883 pazienti sottoposti a trattamento emodialitico e affetti da iperparatiroidismo secondario severo-moderato (la mediana del PTH sierico era di $693 \mathrm{pg} / \mathrm{mL}$ ) (26). Lo scopo primario era di paragonare gli eventi cardiovascolari fatali o non fatali nei pazienti trattati con cinacalcet $(30-180 \mathrm{mg} / \mathrm{die}$, mediana $55 \mathrm{mg} / \mathrm{die}$ ) rispetto a quelli che assumevano placebo. Il follow-up dei pazienti è durato fino a 5 anni con una mediana di osservazione di 21 mesi nel gruppo trattato con cinacalcet e di 17 mesi nel gruppo placebo. Lo studio era multicentrico e randomizzato e i pazienti continuavano la loro terapia convenzionale, inclusi analoghi della vitamina $\mathrm{D}$, supplementi di calcio e chelanti del fosfato. La percentuale di pazienti trattati con cinacalcet che avevano sviluppato eventi cardiovascolari era pari al $48.2 \%$ mentre era del $49.2 \%$ in chi assumeva il placebo. La differenza non era significativa, considerando la frequenza cumulativa degli eventi (Hazard Ratio $[\mathrm{HR}]=0.95$ ) o dei singoli eventi cardiovascolari (morte, infarto miocardico, scompenso cardiaco, angina instabile, eventi vascolari periferici). Tuttavia, quando l'analisi era aggiustata per le caratteristiche basali dei pazienti (età, diabete, origine, storia di eventi cardiovascolari, età dialitica, prodotto calcio-fosforo, fumo, HDL), il rischio cardiovascolare era significativamente più basso in chi assumeva cinacalcet rispetto al placebo $(\mathrm{HR}=0.88)$. Gli Autori hanno valutato i risultati dello studio anche dopo aver prolungato il follow-up di sei mesi: a questo punto di rilevazione, i pazienti trattati con cinacalcet dimostravano una riduzione significativa del numero di eventi cumulativi $(\mathrm{HR}=0.85)$, morte $(\mathrm{HR}=0.83)$ e scompenso cardiaco $(\mathrm{HR}=0.72)$. In sostanza, quindi, applicando rigorosamente $\mathrm{i}$ criteri del protocollo, lo studio EVOLVE non ha dimostrato un ridotto rischio cardiovascolare nei pazienti che assumevano cinacalcet, così come riportato nelle conclusioni dell'articolo, ma, nello stesso tempo, ha dato al lettore una prospettiva positiva sulle potenzialità di protezione cardiovascolare della terapia con cinacalcet (26). È probabile che la difficoltà di ottenere risultati positivi sia dovuta al fatto che lo studio EVOLVE ha considerato i pazienti dializzati, ovvero i pazienti già segnati dalla compromissione vascolare il cui rischio di eventi acuti difficilmente poteva essere modificato dalle terapie.

Lo studio ADVANCE ha, invece, analizzato la progressione della calcificazione vascolare nei pazienti dializzati con iperparatiroidismo secondario, confrontando i pazienti trattati con $1.25 \mathrm{OH}_{2} \mathrm{D}$ o analoghi della vitamina $\mathrm{D}$ con quelli trattati con cinacalcet insieme a $1.25 \mathrm{OH}_{2} \mathrm{D}$ o analoghi della vitamina $\mathrm{D}$ (27). Lo studio era prospettico, randomizzato e controllato. Sono stati arruolati 360 pazienti seguiti da vari centri di nefrologia e sottoposti a emodialisi da almeno 3 mesi. Le calcificazioni erano misurate a livello aortico, coronarico e valvolare 
cardiaco mediante tomografia ed erano nuovamente misurate dopo 52 settimane dalla misura basale. Il volume della calcificazione coronarica aumentava mediamente del $22 \%$ nei pazienti in terapia con cinacalcet e vitamina $\mathrm{D}$ e del $30 \%$ nei pazienti in terapia con la sola vitamina D. La differenza trai due gruppi era significativa. Altre differenze significative di calcificazione erano osservate alla valvola aortica.

Prima di questi due studi soltanto un lavoro aveva analizzato l'effetto clinico cardiovascolare del cinacalcet (28). Si trattava di uno studio su 1184 pazienti emodializzati con PTH superiore a $300 \mathrm{pg} / \mathrm{mL}$, che raccoglieva i dati di quattro studi randomizzati: 697 pazienti erano in terapia con cinacalcet e 487 non assumevano cinacalcet. Nel corso dei 12 mesi di follow-up, il numero di ricoveri era significativamente più basso nei pazienti in terapia con cinacalcet: sono stati osservati 15 ricoveri per motivi cardiovascolari ogni 100 pazienti-anno tra i pazienti che assumevano cinacalcet e 19.7 ricoveri/100 pazientianno tra coloro che non assumevano cinacalcet $(\mathrm{HR}=0.61)$.

\section{CaSR e malattia ossea nella CKD}

Lo studio EVOLVE ha analizzato diversi aspetti clinici potenzialmente influenzati dalla terapia con cinacalcet. Il numero di paratiroidectomie è risultato significativamente più basso nei pazienti in terapia con cinacalcet rispetto al placebo $(2.4 \%$ vs $7.6 \%, \mathrm{HR}=0.44$ ). Questo risultato è la ovvia conseguenza del controllo esercitato dal cinacalcet sulla secrezione del PTH e sulla proliferazione delle cellule paratiroidee (26). Un risultato analogo era stato osservato in un precedente studio, nel quale è stata osservata anche una riduzione del numero delle fratture associata al cinacalcet: esse erano 3.2 per 100 pazienti-anno tra i pazienti trattati con cinacalcet e 6.9 per 100 pazienti/anno tra chi non assumeva cinacalcet (28). Un effetto favorevole sulle fratture non è stato, viceversa, rilevato dallo studio EVOLVE, nel quale il $12 \%$ dei pazienti in terapia con cinacalcet e il $13 \%$ dei pazienti trattati con placebo andavano incontro a una frattura vertebrale o non vertebrale $(\mathrm{HR}=0.89)$. Sono disponibili scarsi dati istologici ossei nei pazienti in terapia con cinacalcet. Un solo studio prospettico in doppio cieco e controllato è stato condotto in pazienti emodializzati con iperparatiroidismo secondario trattati con cinacalcet $(n=19$, dose $30-180 \mathrm{mg} /$ die) o placebo $(\mathrm{n}=13)$ (29). Le biopsie erano eseguite prima del trattamento e dopo 1 anno. La stimolazione del CaSR con cinacalcet riduceva nel siero il PTH e la fosfatasi alcalina ossea. L'esame istologico mostrava una riduzione del turnover, del bone-formation-rate, del numero di osteoblasti e della superficie fibrosa ossea. In tre pazienti trattati con cinacalcet è stata osservata l'insorgenza di osso adinamico (due di essi avevano il PTH sierico inferiore a 100 $\mathrm{pg} / \mathrm{mL}$ a fine studio). La mineralizzazione ossea, viceversa, restava invariata dopo 1 anno di terapia. Questi risultati dimostrano che l'assunzione di cinacalcet modifica il turnover osseo in modo correlato alla riduzione del PTH, probabilmente accrescendo anche il rischio di adinamia ossea. Altri due studi di piccole dimensioni, in pazienti trapiantati di rene e in dializzati, confermano sostanzialmente queste osservazioni istomorfometriche $(30,31)$.

La densità minerale ossea (BMD) è un esame poco usato nella routine dei pazienti con $\mathrm{CKD}$, perché non rispecchia uno specifico quadro istologico osseo e clinico. I suoi valori possono però modificarsi come esito delle variazioni del turnover osseo. Buona parte dei lavori sviluppati nei pazienti con CKD ha osservato un aumento della BMD al collo femorale e al radio dopo un anno di terapia con cinacalcet. L'aumento si correlava all'inibizione dell'attività paratiroidea $(32,33)$. I dati della sede radiale dimostravano che l'aumento della BMD prevaleva all'osso corticale rispetto al trabecolare (33). È stata, però, osservata anche la diminuzione della BMD dopo cinacalcet, a dimostrare la complessità del significato della valutazione densitometrica nel paziente nefropatico (34).

\section{Conclusioni}

Quanto descritto sin qui dimostra l'interesse che l'ambiente scientifico e clinico ha riservato al CaSR e ai farmaci che ne modulano l'attività. Gli studi più recenti hanno, così, rivelato nuove vie che trasducono nella cellula il segnale extracellulare mediato dal calcio, mentre si è ormai affermata la terapia con calciomimetici per l'iperparatiroidismo. Viceversa, ha sostanzialmente deluso le aspettative il ronacaleret, unico calciolitico usato senza successo per l'osteoporosi in studi di terza fase. Per quanto riguarda la CKD, gli studi hanno prestato particolare attenzione agli effetti del cinacalcet sulla malattia cardiovascolare, un argomento che è destinato ad attrarre l'attenzione dei ricercatori anche nei prossimi anni. A tale riguardo, gli studi EVOLVE e ADVANCE hanno dimostrato come può essere difficile ottenere risultati positivi dall'analisi dei pazienti dializzati, le cui condizioni vascolare e cardiaca sono spesso già compromesse. Potrebbe, perciò, essere più utile studiare i pazienti con stadi più precoci di CKD per capire l'efficacia del cinacalcet nella prevenzione della vasculopatia calcifica. Questa conclusione può valere anche per la malattia ossea della CKD, sulla quale sono stati osservati gli effetti positivi del cinacalcet, secondari all'inibizione della secrezione del PTH e all'inibizione del turnover osseo. Non è, però, escluso che i calciomimetici o i calciolitici possano influenzare il metabolismo osseo anche nei pazienti con normali livelli di PTH, in considerazione della loro capacità di influire sull'attività osteoblastica. Tuttavia, per poterne consentire l'uso, oltre a proseguire gli studi sul ruolo del CaSR nel tessuto osseo, bisognerà risolvere il problema del loro effetto sulla calcemia, che oggi ne limita prepotentemente l'applicazione clinica al di fuori dell'iperparatiroidismo primitivo o secondario alla CKD.

\section{Riassunto}

Le cellule dell'organismo umano sono provviste di un recettore per il calcio extracellulare (CaSR) la cui espressione tissutale è ridotta nei pazienti nefropatici cronici. Esso ha un ruolo chiave nella regolazione della secrezione del PTH da parte delle cellule paratiroidee e del riassorbimento del calcio da parte delle cellule tubulari renali. Recenti studi biochimico-cellulari hanno osservato che questo 
recettore ha peculiari caratteristiche funzionali, mentre la ricerca farmacologica ha messo a disposizione farmaci agonisti dell'attività del CaSR (calciomimetici), che sono oggi usati per la cura dell'iperparatiroidismo secondario e che possono avere effetti sulla malattia cardiovascolare e ossea nel paziente nefropatico cronico. A tale riguardo, gli studi EVOLVE e ADVANCE sono stati dedicati a capire il ruolo del calciomimetico cinacalcet nella prevenzione dello sviluppo delle calcificazioni arteriose e degli eventi cardiovascolari nei pazienti nefropatici. Promettenti prospettive sono, perciò, state aperte dalle ricerche in questo recettore e per il trattamento dei malati nefropatici cronici.

Parole chiave: Recettore sensibile al calcio, Iperparatiroidismo

Dichiarazione di conflitto di interesse: Gli Autori dichiarano di non avere conflitto di interessi.
Indirizzo degli Autori:

Dr. Giuseppe Vezzoli

Unità di Nefrologia e Dialisi

Istituto Scientifico Universitario San Raffaele

Via Olgettina 60

20142 Milano

vezzoli.giuseppe@hsr.it

\section{Bibliografia}

1. Hofer A, Brown EM. Extracellular calcium sensing and signaling. Nature 2003; 4: 530-8.

2. Brown EM, Gamba G, Riccardi D, et al. Cloning and characterization of an extracellular Ca-sensing receptor from bovine parathyroid. Nature 1993; 366: 575-80.

3. Riccardi D, Brown EM. Physiology and pathophysiology of the calcium-sensing receptor in the kidney. Am J Physiol Renal Physiol 2010; 298: F485-99.

4. Huang C, Miller RT. The calcium-sensing receptor and its interacting proteins. J Cell Mol Med 2007; 11: 923-34.

5. Thiel G, Lesch A, Keim A. Transcriptional response to calciumsensing receptor stimulation. Endocrinology 2012; 153: 4716-28.

6. Grant MP, Stepanchick A, Cavanaugh A, Breitwieser GE. Agonist-driven maturation and plasma membrane insertion of calcium-sensing receptors dynamically control signal amplitude. Sci Signal 2011; 4: ra78.

7. Breitwieser GE. The intimate link between calcium sensing receptor trafficking and signaling: implications for disorders of calcium homeostasis. Endocrinology 2012; 26: 1482-95.

8. Loupy A, Ramakrishnan SK, Wootla B, et al. PTH-independent regulation of blood calcium concentration by the calcium-sensing receptor. J Clin Invest 2012; 122: 3355-67.

9. Vezzoli G, Soldati L, Gambaro G. Roles of calcium-sensing receptor (CaSR) in renal mineral ion transport. Curr Pharm Biotechnol 2009; 10: 302-10.

10. Gong Y, Renigunta V, Himmerkus N, et al. Claudin-14 regulates renal Ca.. transport in response to CaSR signalling via a novel microRNA pathway. EMBO J 2012; 31: 1999-2012.

11. Toka HR, Al-Romaih K, Koshy JM, et al. Deficiency of the calcium-sensing receptor in the kidney causes parathyroid hormone-independent hypocalciuria. J Am Soc Nephrol 2012; 23 : 1879-90.
12. Chang W, Tu C, Chen TH, Bikle D, Shoback D. The extracellular calcium-sensing receptor (CaSR) is a critical modulator of skeletal development. Sci Signal 2008; 1 (35): ra1.

13. Dvorak-Ewell MM, Chen TH, Liang N, et al. Osteoblast extracellular Ca-sensing receptor regulates bone development, mineralization, and turnover. J Bone Min Res 2011; 26: 2935-47.

14. Gowen M, Stroup GB, Dodds RA, et al. Antagonizing the parathyroid calcium receptor stimulates parathyroid hormone secretion and bone formation in osteopenic rats. J Clin Invest 2000; 105:1595-604.

15. Fitzpatrick LA, Dabrowski CE, Cicconetti G, et al. The effects of ronacaleret, a calcium-sensing receptor antagonist, on bone mineral density and biochemical markers of bone turnover in postmenopausal women with low bone mineral density. J Clin Endocrinol Metab 2011; 96: 2441-9.

16. Fitzpatrick LA, Dabrowski CE, Cicconetti G, et al. Ronacaleret, a calcium-sensing receptor antagonist, increases trabecular but not cortical bone in postmenopausal women. J Bone Min Res 2012; 27: 255-62.

17. Caltabiano S, Dollery CT, Hossain M, et al. Characterization of the effect of chronic administration of a calcium-sensing receptor antagonist, ronacaleret, on renal calcium excretion and serum calcium in postmenopausal women. Bone 2013; 56: 154-62.

18. Kidney disease-improving global outcomes (KDIGO) CKDMBD work group. KDIGO clinical practice guideline for the diagnosis, evaluation, prevention, and treatment of chronic kidney disease-mineral and bone disorder (CKD-MBD). Kidney Int Suppl 2009; 113: S1-130.

19. Canaff L, Hendy GN. Human calcium-sensing receptor gene. J Biol Chem 2002; 277: 30337-50.

20. Awumey EM, Bridges LE, Williams CL, Diz DI. Nitric-oxide synthase knockout modulates Ca-sensing receptor expression and signaling in mouse mesenteric arteries. J Pharm Exp Ther 
2013; 346: 38-47.

21. Bukoski RD. Dietary calcium and blood pressure: evidence that Ca-sensing receptor activated sensory nerve dilator activity couples changes in interstitial ca with vascular tone. Nephrol Dial Transplant 2001; 16: 218-21

22. Lopez I, Aguilera-Tejero E, Mendoza FJ, et al. Calcimimetic R-568 decreases extraosseous calcifications in uremic rats treated with calcitriol. J Am Soc Nephrol 2006; 17: 795-804.

23. Molostvov G, James S, Fletcher S, et al. Extracellular calciumsensing receptor is functionally expressed in human artery. Am J Physiol Renal Physiol 2007; 293: F946-55.

24. Kawata T, Nagano N, Obi M, et al. Cinacalcet suppresses calcification of the aorta and heart in uremic rats. Kidney Int 2008; 74: 1270-7.

25. Koleganova N, Piecha G, Ritz E, Schmitt CP, Gross ML. A calcimimetic (R-568), but not calcitriol, prevents vascular remodeling in uremia. Kidney Int 2009; 75: 60-71.

26. Chertow GM, Block GA, Correa-Rotter R, et al. Effect of cinacalcet on cardiovascular disease in patients undergoing dialysis. N Engl J Med 2012; 367: 2482-94.

27. Raggi P, Chertow GM, Torres PU, et al. The ADVANCE study: a randomized study to evaluate the effects of cinacalcet plus low-dose vitamin $\mathrm{D}$ on vascular calcification in patients on hemodialysis. Nephrol Dial Transplant 2011; 26: 1327-39.

28. Cunningham J, Danese M, Olson K, Klassen P, Chertow GM. Effects of the calcimimetic cinacalcet $\mathrm{HCl}$ on cardiovascular disease, fracture, and health-related quality of life in secondary hyperparathyroidism. Kidney Int. 2005; 68: 1793-800.

29. Malluche HH, Monier-Faugere MC, Wang G, et al. An assessment of cinacalcet $\mathrm{HCl}$ effects on bone histology in dialysis patients with secondary hyperparathyroidism. Clin Nephrol 2008; 69: 269-78.

30. Borchhardt KA, Diarra D, Sulzbacher I, Benesch T, Haas M, Sunder-Plassmann G. Cinacalcet decreases bone formation rate in hypercalcemic hyperparathyroidism after kidney transplantation. Am J Nephrol 2010; 31: 482-9.

31. Yajima A, Akizawa T, Tsukamoto Y, Kurihara S, Ito A. Impact of cinacalcet hydrochloride on bone histology in patients with secondary hyperparathyroidism. Ther Apher Dial 2008; 12 (Suppl. 1): S38-43.

32. Tsuruta Y, Okano K, Kikuchi K, Tsuruta Y, Akiba T, Nitta K. Effects of cinacalcet on bone mineral density and bone markers in hemodialysis patients with secondary hyperparathyroidism. Clin Exp Nephrol 2013; 17: 120-6.

33. Ishimura $\mathrm{E}$, Okuno $\mathrm{S}$, Tsuboniwa $\mathrm{N}$, et al. Effect of cinacalcet on bone mineral density of the radius in hemodialysis patients with secondary hyperparathyroidism. Clin Nephrol 2011; 76: 259-65.

34. Mitsopoulos E, Ginikopoulou E, Economidou D, et al. Impact of long-term cinacalcet, ibandronate or teriparatide therapy on bone mineral density of hemodialysis patients: a pilot study. Am J Nephrol 2012; 36: 238-44. 